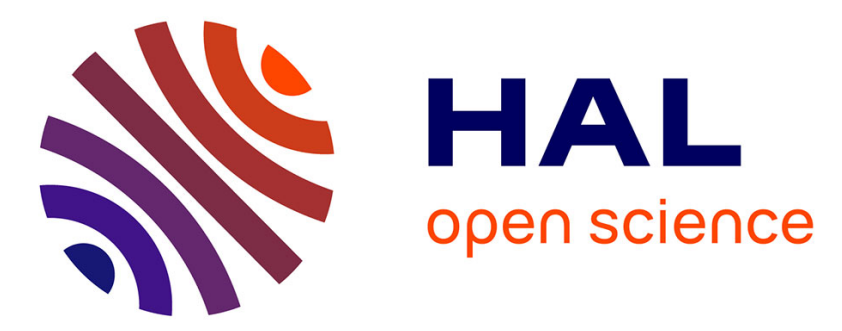

\title{
E-cooperative design among mechanical and electrical engineers: implications for communication between professional cultures
}

Benoît Delinchant, Vincent Riboulet, Laurent Gerbaud, Philippe René Marin, Frédéric Noël, Frédéric Wurtz

\section{To cite this version:}

Benoît Delinchant, Vincent Riboulet, Laurent Gerbaud, Philippe René Marin, Frédéric Noël, et al.. E-cooperative design among mechanical and electrical engineers: implications for communication between professional cultures. IEEE Transactions on Professional Communication, Dec 2002, -, France. pp.231 - 249. hal-00333844

\section{HAL Id: hal-00333844 https://hal.science/hal-00333844}

Submitted on 24 Oct 2008

HAL is a multi-disciplinary open access archive for the deposit and dissemination of scientific research documents, whether they are published or not. The documents may come from teaching and research institutions in France or abroad, or from public or private research centers.
L'archive ouverte pluridisciplinaire HAL, est destinée au dépôt et à la diffusion de documents scientifiques de niveau recherche, publiés ou non, émanant des établissements d'enseignement et de recherche français ou étrangers, des laboratoires publics ou privés. 


\title{
E-Cooperative Design Among Mechanical and Electrical Engineers: Implications for Communication Between Professional Cultures
}

\author{
-BENOIT DELINCHANT, \\ VINCENT RIBOULET, \\ LAURENT GERBAUD, \\ PHILIPPE MARIN, \\ FRÉDÉRIC NOËL, \\ AND FRÉDÉRIC WURTZ
}

\begin{abstract}
This paper looks at the collaborative design activity involved in a design experiment of an electromechanical plunger. Much of the coordination was achieved through internet-based communication. As mechanical and electrical researchers involved in the design project, we discuss the information exchanges highlighted by our different professional cultures and relate how these exchanges lead us to propose some methodology to improve the efficiency of virtual meetings. Moreover, we show the need for new communication tools, ones dedicated to specific tasks that are not currently supported, especially shared concept formalization among technical experts.
\end{abstract}

Index Terms-Collaborative design, electrical and mechanical engineering, internet-based information exchange, professional culture, shared objects, virtual teams.

Manuscript received February 27, 2002; revised June 17, 2002.

B. Delinchant, L. Gerbaud, and F. Wurtz are with the

Laboratoire d'Electrotechnique

de Grenoble (LEG),

UMR-5529 CNRS-INPG/UJF,

EMSIEG, BP46,

F-38402 Saint Martin d'Héres Cèdex,

France

(email: Benoit.Delinchant@

leg.ensieg.inpg.fr;

Laurent.Gerbaud@leg.ensieg.inpg.fr.;

Frederic.Wurtz@leg.ensieg.inpg.fr).

P. Marin, F. Noël, and V. Riboulet

are with the Laboratory

"Sols-Solides-Structures" (3S).

UMR-5521 UJF-INPG-CNRS, BP 53,

38041 Grenoble Cedex 9, France

(email: Phillipe.Marin@inpg.fr;

Frederic.Noel@inpg.fr;

vincent.riboulet@inpg.fr).

IEEE DOI 10.1109/TPC.2002.805149 ncreasingly, the design of technical products tends to be a collaborative activity in which concurrent engineering methodologies are developed in order to involve the different points of view on the product, all along its life cycle. In order to increase information exchange efficiency and to ensure the best integration of the different experts involved in the design process, new tools and new methodologies have been proposed. Our research deals with these concurrent engineering and collaborative work approaches.

Design activity often requires the collaboration of designers from different skills and from different professional cultures. This trend is pushed by the evolution of the products becoming more and more complex, thus necessitating optimization from any point of view. In parallel, the globalization of industrial activity and decentralization of many manufacturing processes lead companies to work in relation to very distant collaborators.

The expansion of internet-based tools has opened new opportunities for collaborative work improvement. Our research focuses on the feature specification and development of such tools.

In this paper, we first present a literature survey to situate our study in the field of collaborative design. We discuss the available tools supporting collaborative work over the internet in an industrial context. We address electronic 
collaboration in the context of the negotiation between the professional culture of mechanical and electrical engineers. Finally, we detail the contemporary solutions for collaborative work.

In the next section, we detail the collaborative experiments we have made. The hypothesis as well as the chosen design example (an electromechanical plunger: EMP) are presented. We detail the different kinds of experiments with their objectives, and we focus on two technical aspects which have to be solved thanks to a confrontation between the cultures of the electrical and mechanical engineers.

We then discuss and present the results of this work that help answer the following questions:

- What are the communication typologies (formal, informal, synchronous, asynchronous) and the tools able to support them?

- What kinds of different meetings appear?

- Which information is exchanged, what are the concepts and the new requirements of a tool able to support such exchange?

Finally, we present a framework for the new tools we started to develop. They are dedicated to support complementary aspects of communication in a multiexpert design activity over the internet, providing communication features not available in present-day commercial solutions.

\section{LITERATURE SURVEY AND WORKING CONTEXT}

\section{Industrial Context: CAD or} PDM to Share Product Data The

challenge for software developers is to provide users with solutions that allow the best compromise between communication among themselves, and ease of use in his or her daily activity. The commercial solutions commonly developed in the CAD (computer-aided design) context for several years comprise two categories.
The first category requires every participant in the design process to work around the same CAD/CAM (computer-aided manufacturing) software and to provide lots of "expertise" modules in that software (geometric modeling, various simulations, different manufacturing or assembly processes, drawing and billing of material edition, etc.), in an attempt to cover the needs of each participant. This is the choice of tools such as Pro/Engineer, Catia, Unigraphics, Ideas, etc. In this way, everybody in the project is working around the same geometric model [1], and there is no need of information translation, or if there is such a need, the translation is provided by the expert module itself.

The second category allows users to work with their preferred specific tools but also imposes a common PDM (product data management) software such as Agile, Windchill, Enovia, Pro/PDM. The goal is to ensure that the available data is always unique, up to date (maybe keeping a history of versions), and to avoid the need of asynchronous data transfers. The collaboration between the participants is organized through the PDM system sharing computer files representing the product. As in the previous category of solutions, this approach does not provide synchronous collaboration. Moreover, it does not avoid translations between expert tools, through the various standard file formats for three-dimensional (3-D) geometry (IGES, SET, VDA, STEP, etc.). One can also note that there is a lack of standard exchange formats for other kinds of information.

In this paper, we show that there is actually a need to develop new software tools that will support synchronous and asynchronous dialog between technical experts, providing them with the ability to inter-trade a formalization of shared concepts.
Cultural Aspects Our research project focuses on collaboration among mechanical and electrical engineers, who have different scientific and technical cultures, their own specific semantic fields [2], different ways to solve design problems, and different simulation tools that do not manipulate similar concepts, information, or parameters. As studies of cultural aspects propose [3], one may distinguish several aspects: national culture [4], corporate culture, professional culture, and branch culture. In our experiment, national culture is the same for everybody (it is not a factor influencing the design process or the exchange of information like in [5]). Branch culture is not relevant, and corporate culture is not supposed to be a major factor (this could be discussed as all the designers are researchers, thus having probably some common values). This said, the major difference between the two teams is professional culture.

\section{A Mechanical Point of View: Our} perception of the mechanical design culture is that it is mainly organized around tasks shared among several participants, each competent in a specific subfield. In this context, each designer often has specific goals, manipulates specific tools and knowledge, and may appear to belong to a specific cultural domain [3]. With this in mind, one can also refer to the concept of "worlds" as defined by Mer [6]. This concept is based on three sociological notions.

- The ACTION LOGIC, taken from [7], which relates to goals and issues of involved people and to the action itself.

- The scale of VAlue, taken from [8], which leads to some rules to evaluate a technical solution with respect to various criteria, and which is used especially to justify the action.

- The COLLECTIVE KNOWLEDGE, which refers to shared knowledge, along with 
conventions, implicit or explicit rules manipulated by people in that world.

While, from the analysis of a particular company, Mer proposes to distinguish the scientific world, the industrial world, the customer world, and the buyer world, one can speak in our case about the designer world, the simulation world, and the manufacturing world. Each of them has its own action logic, collective knowledge, and scale of value. In sum, they are of different professional cultures.

Let us add that the way mechanical engineers are designing has consisted for several decades in combining standard technological components with some kinds of structural innovations. They also managed loop simulations for choosing material, dimension, manufacturing, strength, or behavior, until they can get a satisfactory product definition. This way of doing implies intensive exchanges between involved actors. This is a special feature of the mechanical engineering culture.

\section{The Electrical Engineers' Point of} View: From our point of view, when designing electrical devices like a motor, a transformer, or a plunger, the designer must have a global vision of the device for the following reasons:

- Electromagnetic phenomenon is linked to the thermal and mechanical phenomena. For example, the current in the windings or the magnetic flux in the iron parts generates losses that are at the origin of the thermal phenomenon, which, in turn, changes some electromagnetic characteristics like the resistance of the windings. In the same way, the mechanical constraints and limits must be known since the possible values of the air gaps or the section of the magnetic materials (like iron) depend on them.

\section{- The electromagnetic phenomenon propagates in all the devices around (theoretically in all the space).}

This necessity of a global vision explains why electrical designers often work alone or in a small team. The electrical designer works with simulation tools that help portray various physical phenomena. This is a special feature of electrical design culture.

In spite of this traditional way of working, it has become apparent in the last few years, that devices are becoming more and more compact and complex. Their electrical design requires very specific skills in fields such as power electronics or electromagnetism, skills that are distributed between several specialists. Thus, collaborative design processes must be developed and improved.

\section{Industrial Design Process of} Electromechanical Products: Let us point out that in most electrical companies, in the recent past, electrical devices were still designed in two distinct steps (design of magnetic parts without involving significant dialog with other engineers, and then passing the file to mechanical and manufacturing offices). This activity tends to become more collaborative, covering the whole process, in order to get optimized in a global point of view. But there is a lack of computer communication tools able to support the required interprofessional technical exchanges.

In fact, an important part of the design process consists in exchanges between participants of different professional cultures (for instance, electrical and mechanical engineers). Even in each domain, different professional cultures and specialists need to interact. Of course, those processes already exist, but they have to be improved.

\section{Present-Day Solutions for Collaborative Work: ICT What}

are the possibilities of ICT (internet communication tools) as a communication media? We surveyed available technologies, in terms of tools that allow collaborative work over the internet.

Communication Tools: The first category of software concerns classic communication needs. Some of these tools are now widespread.

Some tools allow technical structured data to be shared or transferred (draft sketches or precise geometric definition, 2-D or 3-D, etc.) while others are mostly dedicated to exchange nonstructured information.

According to van Luxemburg and Ulijn [9], these differences in media richness depend on the number of social clues that can be transported by the medium. Hence, choosing a good media depends on the amount of explicit information used in the activity. This leads the authors of [9] to propose an interesting classification of media richness (see Fig. 1).

Relatively little software can put several tools together in a collaborative environment, covering a wide panel of the scale of Fig. 1. Let us cite Microsoft NetMeeting, HP Shared-X, and SGI Inperson. The last provides the ability of sharing 3-D CAD models in the whiteboard, which can be very useful in mechanical part design activity.

Dedicated Tools: A new category of tools has appeared recently that are specifically dedicated to collaborative technical-product design over the internet. These tools offer a team of engineers (including design, production or marketing offices, subcontractors, etc.) a way of working together on a technical activity, sharing data about the product in question [10] [11].

The functionalities of commercially available tools of this kind have been classified (see Table I). As 
one can see, most of these tools functionalities are an integration of several communication tools and a PDM, complete with the ability of visualizing a common 3-D model, and sometimes to modify it.

These solutions cannot support a satisfactory technical communication among a design team, if several engineers of different professional cultures have to collaborate from different places, and especially with different tools matching their own needs and practices. For example, the main function of these dedicated tools, 3-D sharing, is not appropriate for electrical engineers who do not use a 3-D model as a working base.

Organization Tools: Industry clearly asks for some organization and working methodologies in order to manage their distributed design activity. In particular, two different aspects are discussed below: workflow management during the whole design project and synchronous meetings organization.

Presently, PDM software include project and workflow management facilities. They are evolving toward a "web-based" model, providing easy accessibility through a simple browser to the main functionalities (PTC Windchill, EDS Team Center, Dassault Systems Enovia Portal). Their area of concern is expanding with the PLM concept (Product Lifecycle Management): the idea here is to integrate every participant of the whole design activity, including, for example, subcontractors or even customers.

But these widespread industrial tools have two limitations. First, they are based on predefined workflow definitions. Some research teams are working on these topics. Let us cite works of the APEL research project [12] in the computer science field which tries to bring tools to manage the workflow when a conflict appears during creation of software.

Second, there is no organization tool dedicated to provide synchronous meeting management facilities. Recent research [13] tries to give directions for an effective leadership of virtual teams by closely analyzing their typology according to criteria such as temporal distribution (synchronous or not), boundary spanning (in terms of mixing different cultures, for example), the life cycle of the team (discrete or continuous), and the variety of member roles.

\section{A Concept for Sharing Knowledge: Intermediary Objects Among the analyzed} tools no solution can dynamically

Fig. 1. Media richness in a qualitative scale.

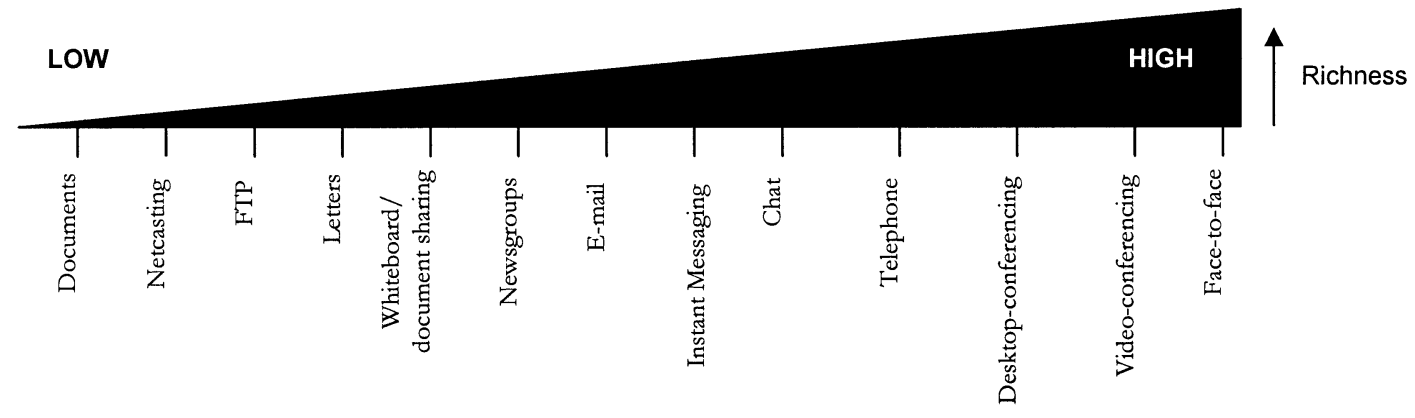

TABLE I

AN Overview OF COMMERCIAL COLlaborative

DESIGN TOOLS [11]

\begin{tabular}{|c|c|c|c|c|c|c|c|c|c|c|c|c|c|c|c|c|}
\hline \multirow[b]{2}{*}{ Tools } & \multicolumn{10}{|c|}{ Technical Communication } & \multicolumn{3}{|c|}{ Documents } & \multicolumn{3}{|c|}{$\begin{array}{c}\text { Project } \\
\text { Management }\end{array}$} \\
\hline & 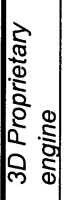 & 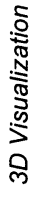 & 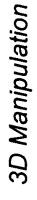 & 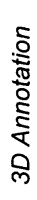 & $\frac{\pi}{0}$ & $\frac{\xi}{5}$ & 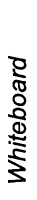 & 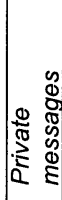 & 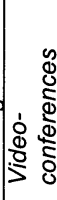 & 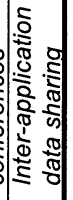 & 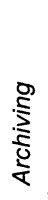 & $\begin{array}{l}\frac{0}{5} \\
\frac{0}{5} \\
\frac{0}{0} \\
\end{array}$ & 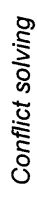 & 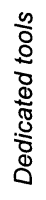 & 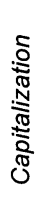 & 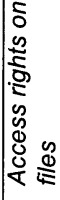 \\
\hline One space & & $\mathrm{X}$ & & $\mathrm{X}$ & $\mathrm{X}$ & & $x$ & $\mathrm{x}$ & & & & & & & & $\mathrm{X}$ \\
\hline Centric & $\mathrm{X}$ & $\mathrm{X}$ & $\mathrm{X}$ & $\mathrm{X}$ & $\mathrm{X}$ & $\mathrm{X}$ & & $\mathrm{X}$ & $\mathrm{X}$ & & $\mathrm{X}$ & $\mathrm{X}$ & $x$ & $\mathrm{X}$ & $\mathrm{x}$ & $\mathrm{X}$ \\
\hline Alibre & $\mathrm{X}$ & $\mathrm{x}$ & $\mathrm{X}$ & & $\mathrm{x}$ & $\mathrm{X}$ & & $x$ & & & $\mathrm{X}$ & $\mathrm{x}$ & $\mathrm{X}$ & $\mathrm{X}$ & & $x$ \\
\hline Reality wave & $\mathrm{X}$ & $\mathrm{X}$ & $x$ & $\mathrm{X}$ & & & & $\mathrm{X}$ & & & & & & & & \\
\hline Groove & & $\mathrm{x}$ & $\mathrm{X}$ & $\mathrm{X}$ & $\mathrm{X}$ & & & $\mathrm{x}$ & & & & $\mathrm{X}$ & $\mathrm{X}$ & $\mathrm{X}$ & $x$ & $\mathrm{x}$ \\
\hline$E$-vis & & $\mathrm{X}$ & & $\mathrm{X}$ & $\mathrm{X}$ & $\mathrm{X}$ & $\mathrm{X}$ & $\mathrm{X}$ & & & $\mathrm{X}$ & $\mathrm{X}$ & $\mathrm{X}$ & $\mathrm{X}$ & & $\mathrm{X}$ \\
\hline Informative Graphics & & $x$ & & & & $\mathrm{X}$ & & & & & $\mathrm{X}$ & & & & & \\
\hline Nexprise & & $x$ & & $x$ & $x$ & & & & $x$ & & $x$ & $x$ & $x$ & $x$ & $x$ & $x$ \\
\hline $\mathrm{Co}$ & & & & & & & & & & $x$ & & & & & & \\
\hline
\end{tabular}


support the definition of a new object or concept defined from the dialog of two engineers in the flow of their daily work.

That is why we state positively that focusing on communication between software [14] and direct exchanges of predefined concepts cannot lead to efficient communication between craftsmen or every participant in the design process. Thus, we turn toward communication between people who are familiar with the project context, can explain their specific concepts and parameters to each other, and then define and dynamically format shared entities. This approach is based on the concept of INTERMEDIARY OBJECT as used in [15], and as defined in [16] as follows:

We simply use the term intermediary to mean that objects lie in between several elements, several actors, or successive stages of a work process (intermediary results). We shall thus talk about intermediary objects as a general way of designating drawings, files, prototypes, which mark the transition from one stage to another, circulate from one group to another or around which various actors and instruments revolve.

But we also point out the idea that each of these objects must be defined, stored, and manipulated together with its contextual explanation. In this way, every information exchange becomes clear for every actor concerned with it, without misunderstanding, and the translation procedure may be defined by each actor according to their own knowledge and practice.

Thus, on the opposite of the approach used in SCM software (System Configuration Management), where shared objects management is automated in a way based on workflow and predefined objects life cycle [17], [12], we choose to provide a more informal way of creating and managing shared information, so that it may be used either in scheduled collaborations or in opportunistic interactional contexts [18]. With this in mind, we need to look closely at the PACT project developments [19], where a study of some domain-specific engineering ontology is proposed. Nevertheless, we assume that, despite every effort that can be made to define such common engineering artifacts and processes in a given domain of expertise, engineers may always have to define new shared concepts and vocabulary dynamically matching their needs in the collaborative design work. As noted in [20], [21], and [22], the building of a "common information space" in CSCW should not simply consist of objects, events, e.g., in a shared database, but also should involve the joint interpretation of these objects and events by the actors involved:

Cooperative work is not facilitated simply by the provision of a shared database, but requires the active construction by the participants of a common information space where the meanings of the shared objects are debated and resolved, at least locally and temporarily. Objects must thus be interpreted and assigned meaning, meanings that are achieved by specific actors on specific occasions of use.

\section{EXPERIMENTS}

In this section, we will describe the experimentation, over three sections.

The first presents the design experiment framework, with the device to be designed, and the context of work.

The second presents sequentially four collaborative experiments that will be discussed in the next section.

Finally, the third focuses on two specific technical problems that have been treated through the four previous experiments.

\section{Device to be Designed and Outlines of the Design Process}

An Electromechanical Plunger: In this paper, collaborative design is illustrated on an electromechanical plunger (EMP) [23] detailed in the Appendix and shown in Fig. 2.

The design of such a system requires several steps.

- Definition of the structure and choice of material for the different parts, by considering the physical constraints of the process. For example, the magnetic flux has to be considered in some parts but not in other ones, the machining of steel modifies its magnetic properties, etc. In this step, manufacturability is also taken into account.

- Modeling of the physical (electrical and mechanical) aspects of the system by using calculation tools with fine modeling. This task allows us to analyze some phenomena and to have reference models for the creation of simple models dedicated to the sizing.

- Sizing by using optimization techniques if possible.

- Definition of the manufacturing process and assembly planning.

In every step, contradictions between mechanical and electrical aspects appear. For example, the section of the body must be sufficient to assure a magnetic flux density, but also must not be too thick, according to manufacturing process constraints. Thus, many choices have to be made,

Fig. 2. Principle of an elctromechanical plunger.

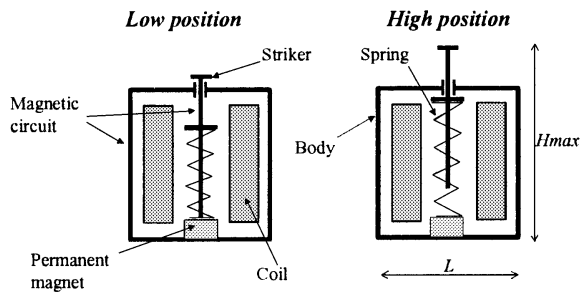


involving several exchanges among the participants of different professions. In this design process, several tools must be used so the designers may exchange several kinds of information. Fig. 3 shows an example of 3-D geometry developed by a mechanical engineer and proposed to the electrical engineers.

The Appendix first develops a technical presentation of the specification of the plunger and then presents the profession tools necessary for its design.

Hypothesis and Context of Working: This paper only deals with collaborative work via internet, hence at distant places. Face-to-face interaction and phone exchanges are not discussed and will not be dealt with in the following experiment descriptions.

\section{Experiments: Collaborative Design Over the Internet To} achieve the design of the electromagnetic plunger, three very different design meetings over the internet have been organized. The study of those meetings allows us to better understand which kind of tools and exchanges are required for a collaborative work over the internet. Each design session had very specific objectives.

Several roles were taken by the participants:

- the project leader who had to coordinate the project review;

- the customer, defining the EMP specifications;

Fig. 3. A view of the plunger 3-D model at an intermediate design stage.

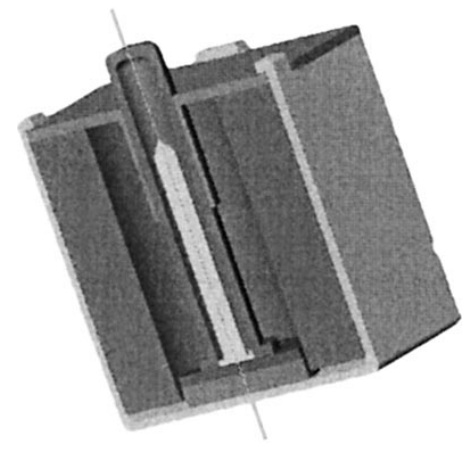

- the mechanical designer;

- the manufacturing engineer;

- the electrical engineer (both for the characterization of the structure and the sizing).

However, before presenting these sessions, we will develop the first phase of our design experiment.

\section{Forum Tool, Supporting}

Asynchronous Work: To start the codesign project, a discussion forum was created for the collaborative choice of the structure of the EMP from specifications defined by the customer. Such a forum was well adapted to support and to capitalize the asynchronous exchanges between actors.

In this way, the forum was used to save files in a technical format:

- Professional drawing tools. Their file format cannot be manipulated by everybody in the design team (e.g., SolidWorks, Pro-Engineer, Flux, Mathcad, Pascosma-Eden, etc.). As the forum allows the exchange of any file types, the actors have naturally chosen to use screen captures to show the structure drafts from these professional tools.

- Other software like Microsoft PowerPoint and Microsoft Paint were used because they offer the opportunity to create drafts and pictures, and they are available for every actor.

- Some designers have drawn their drafts on paper sheets and scanned them. These drafts were often completed by explanations, questions, and remarks (see Fig. 4).

As this exchange mode is too asynchronous and not sufficiently interactive, this experiment has not given very satisfying results.

Definition of the Plunger Structure: The goal of this meeting was to design the plunger's structure, out of the initial specification sheet. The configuration of the meeting was the following:
- on the mechanical side, the engineers were dispatched in two different sites with computers and specific tools, one site for mechanical design, and one for manufacturing analysis;

- on the electrical side, all the engineers were in the same room with one PC.

The used environment was NetMeeting with its chat tool, its tool for application sharing, and its whiteboard. When needed, the teams agreed to synchronize their exchanges using the phone. This possibility was necessary, since NetMeeting only allows participants to use voice exchanges from point to point, hence only between two participants.

As the special functionalities of commercial collaborative dedicated tools were not really interesting in our case (see the next subsection), we decided to use only the nondedicated communication possibilities, such as the ones found in classical and free communication tools described later.

To be able to design a structure for the device, the following kind of information has been exchanged.

- Information about the electrical phenomena that occur in the EMP. As shown on Fig. 4, some drafts on the whiteboard were presented to explain complex phenomena. For

Fig. 4. Example of exchange via the forum: remarks of the electrical engineers on a structure (draft) proposed by a mechanical designer.
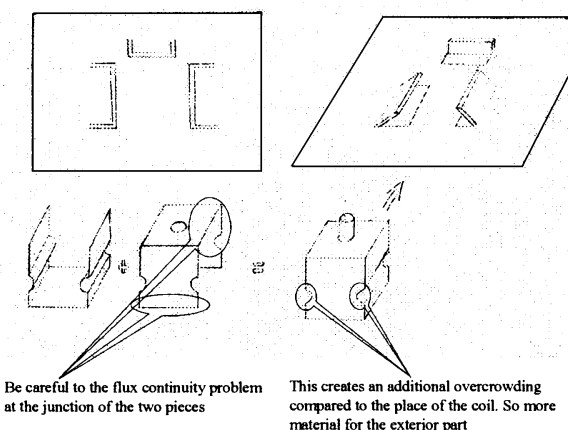
instance, on the sheet of Fig. 5, the electrical engineers explain how the magnetic flux is created and how it propagates in the device. This was also the opportunity to explain to the mechanical engineers what are the critical air gaps for this propagation. Air gaps that should be avoided or minimized in the mechanical structure.

- Information about the structure that the mechanical engineers have proposed. First, the 3-D mechanical geometric modeler (SolidWorks) was shared on one PC throughout NetMeeting. This was not very successful because it was a bit slow over the net. Moreover, electrical engineers were not familiar with this mechanical software interface. Second, drafts were made by hand by the mechanical engineers on the whiteboard (see Fig. 6). Those diagrams gave the opportunity to explain the solution and to discuss the drafts when some problems occurred.

Pretty quickly, this meeting turned into simple exchanges between two actors, in a point-to-point mode. This can be explained by difficulties synchronizing the exchanges and using the whiteboard in a coordinate way. We tried to share an application on one PC, but, as previously said and despite the fast internet connection (10 Mb/s), this was slow and inefficient. This was probably due to "heavy display"

Fig. 5. Sheet presented by the electrical engineers on the whiteboard to explain to the mechanical engineers the path of the magnetic flux.

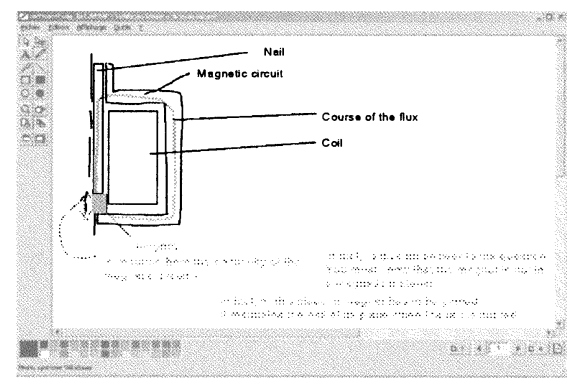

of the 3-D geometric modeling software.

The experiment highlighted some lack of efficiency, but at last, the choice of the structure was made after a few point-to-point exchanges.

Sizing the Structure of the Plunger: For this meeting, Microsoft NetMeeting was used again but along with Paltalk for its multipoint audio facility.

The configuration of the meeting was the following:

- On the mechanical side, the engineers were dispatched in two different rooms again: one in a room with a PC and two participants in another room around one PC.

- On the electrical side, all the engineers were in two rooms (two in each), but each actor had his own PC.

- For this structure sizing, some information must be exchanged, as follows.

- Equations: an example of this is the equation of the force of the spring. One participant in the mechanical community had to design this spring. This design had to be transmitted to the electrical engineers. They had to size the electromagnetic structure to produce a static electromagnetic force able to contain the force

Fig. 6. Manual drawing used to explain and discuss the structure for the magnetic core chosen by the mechanical engineers.

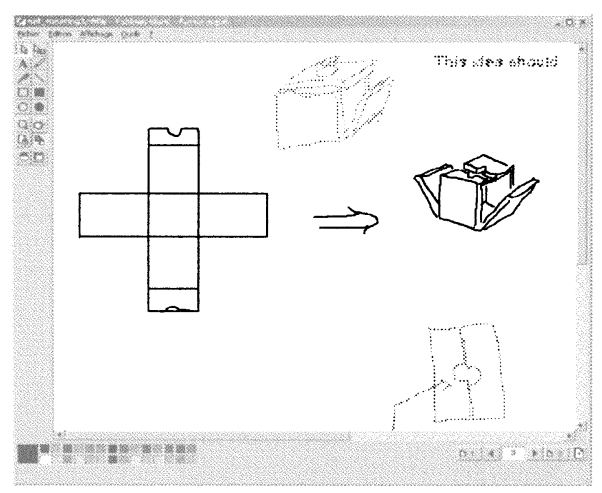

of the spring. The spring and the corresponding equation were discussed during the meeting through oral informal communication as well as through the chat window (see Fig. 7).

- Values given to some parameters that must be explained by one participant to the others as well as the units.

- Meaning of the parameters and the equations.

An efficient media to explain the meaning of some parameters is, of course, the whiteboard. Fig. 8 shows an interesting example of a whiteboard used during the meeting. On the left side, the parameterization of the model used by the electrical engineers to calculate the electromagnetic force is presented. On the right side, a part of the mechanical structure and model designed by the mechanical engineers is shown. By having both views on the whiteboard simultaneously, the participants could link the geometric parameters used in both models and both communities. This sheet could be used to "link" in both models the maximum length of the spring and other related parameters, but this link remained virtual in engineers' minds. There is currently no mean in standard communication tools to link one parameter in a mechanical design software to another parameter in an electrical simulation software. Note that this link, its meaning, and its parameters can be seen as a simple example of common knowledge created dynamically

Fig. 7. An example of the content of the chat window when the discussion was about the equation of the spring.

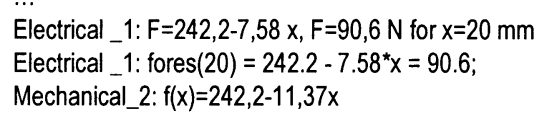


between mechanical and electrical engineers.

A Project Review: The goal of this meeting was a briefing about all the work done for the design of the plunger. As in the previous meeting, Paltalk and NetMeeting were used.

For this project review, each participant had to prepare a talk fitting in a limited timeframe. A moderator was identified in order to synchronize and synthesize all the presentations made.

The meeting began with a presentation of a participant sharing his PowerPoint application throughout the net. This time, the sharing of this "light-display" software was very successful. This was the opportunity to explain again the specifications and mainly the constraints the team encountered. Each member of the team who was involved in the study presented his contribution. The meeting was structured, driven, and very efficient.

\section{Focus on Two Technical}

Aspects As the design of the plunger is a joint problem of mechanics and magnetism, it must result from a close interaction between both of the professions. This section focuses on two discussions among the various undertaken experiments.

Fig. 8. Whiteboard used to compare the electromagnetic model and a part of the mechanical model. It was particularly used to find the maximal length of the spring in both models.

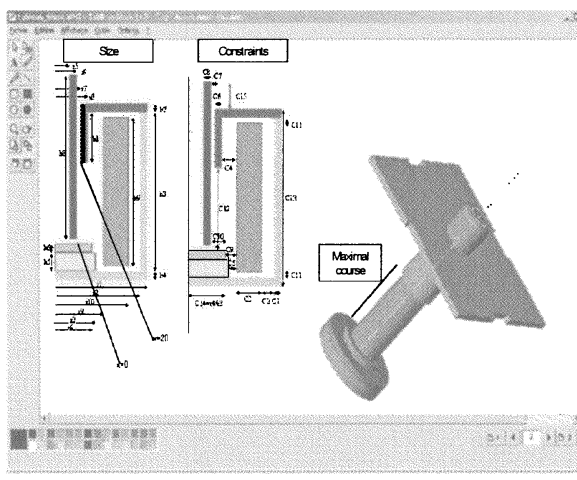

The Magnetic Problem: Air Gap Versus Clearance: The example of the air gap illustrates the interaction of mechanical choices on the magnetic design.

What is the impact of the clearance around the plunger and the slacks in the magnetic circuit that introduce air gaps and, hence, disturbances in the magnetic flux ?

Initially, this aspect appeared during the design of the structure while using the forum, and then in synchronous discussions during online meetings. The forum appeared to be too asynchronous so that a lot of energy was lost by the participants explaining their choices, and it was very time-consuming. So we decided to use synchronous meetings to choose the structure of the EMP.

Problem specifications were given by electrical engineers with the principle draft of Fig. 2. Then, several solutions were proposed by the mechanical actors and discussed by the electrical ones in the forum. Fig. 10 shows an example of such an exchange. Finally, as there were misunderstandings, a videoconference was used to synchronize the knowledge of everybody on the problems.

In this way, participants on the electrical side added remarks on the draft created by the participants on the mechanical side. As this was not sufficient, expert rules were formulated by the electrical engineers to explain some choices. For example, the shape of the EMP envelope is either circular or squared, whenever the section area is sufficient for the crossing of the magnetic flux. Finally, a compromise was found between a small thickness with respect to weight and the cost aspects and a sufficient thickness with respect to the flux crossing area and loads.

In the same way, a compromise was discussed to define the clearance around the plunger's nail without it having a too large air gap. The problem is, for the mechanical engineer, that the clearance around the nail is very important for its movements, but it is obstructing the magnetic circuit because it creates an air gap in the flux trajectory. Both participants had their own view and saw different constraints, so they had to compromise to ensure a good design that respects all manufacturing, functional, and cost constraints.

The Spring Problem: This problem appeared when the team worked on the equilibrium of the plunger. The element involved here is the spring (see Appendix for detailed explanation of how the EMP is working).

During the online sizing meeting, mechanical engineers have given the equation that may represent the spring strength depending on the plunger position. This equation was introduced within the electrical model, but the sizing process did not converge. This divergence was due to unsatisfied constraints, particularly geometrical constraints. In fact, the spring was too strong to be contained in the maximal bounding size allowed by the specification sheet.

Our first sizing try raised some questions about codesign requirements as it constrained the natural designer behavior. After several interchanges between the concerned designers, the following choices were proposed to continue the codesign:

- an iterative process (try and miss);

- an increase in the common knowledge container;

- integration of all knowledge [19], [21].

A natural iterative process would be to ask the mechanical engineers to propose another spring and retry the sizing. But we tried a 
better way of codesigning rather than a simple "try and miss" methodology.

To avoid many back and forth attempts, we tried to increase the common information container by explaining the magnetic constraints on the spring. This allowed the mechanical engineers to have an idea of the spring maximal strength needed. The mechanical engineers could then redesign the spring trying to take into account these new constraints.

\section{RESULtS AND Discussion}

Our choice of public and generic communication tools has shown its possibility to achieve our design goal (Fig. 9) and to support the emergence of a common culture. But it has also led to efficiency limitations, due to some specific communication, organization, and information exchange needs that were poorly supported.

\section{Adapted Tools to Communica- tion Needs In this subsection} we summarize some technical aspects of communication tools used during our experiment. A brief overview of communication modes are recalled, followed by a summary of the ICT used during the study. A short paragraph is then dedicated to tools supporting voice media, and finally, hardware use is discussed.

Fig. 9. Collaboratively designed plunger.

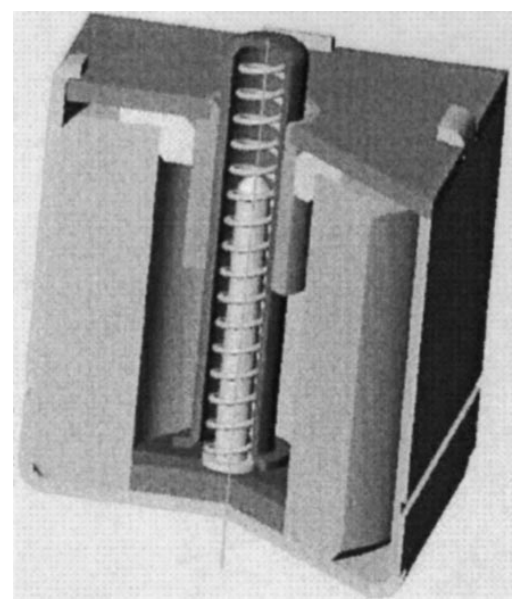

A Typology of Communication Modes: As we have shown in previous sections, information exchanges may be classified depending on their content and use:

- Some are informal and concern discussions which aim to explain, to justify, and to clarify concepts.

- The others are formal, e.g., constraints (the spring force at its high and low positions), data (a geometry with parameters, material properties), design rules, etc.

Information exchanges may also be separated from a time point of view; as already mentioned (see also Table II), there are two communication modes:

- Synchronous mode: instantaneous exchanges between the actors; NetMeeting or Paltalk mainly use this mode.

- Asynchronous mode: this allows to store the knowledge so that it can be accessed at any time by every actor; electronic mail and the discussion forum use this mode.

ICT Use During Our Study: Internet Communication Tools (see Table I) are the support of a virtual team communication. They may be numerous usable tools

TABLE II

Electronic Media, Derived From the SPace/Time Matrix

\begin{tabular}{|c|c|}
\hline $\begin{array}{c}\text { Exchange } \\
\text { Type }\end{array}$ & Media Tools \\
\hline Synchronous & $\begin{array}{ll}- & \text { Chat } \\
\text { - } & \text { Instant Messengers } \\
\text { - } & \text { Desktop } \\
& \text { conferencing } \\
\text { - } & \text { Whiteboard sharing } \\
\text { - } & \text { Document sharing } \\
\end{array}$ \\
\hline Asynchronous & \begin{tabular}{|ll} 
& E-mail \\
- & Newsgroups \\
- & Common data base \\
& sharing \\
- & Repository: CVS \\
& (concurrent version \\
system) or FTP \\
(File Transfer \\
Protocol) \\
- Web publishing
\end{tabular} \\
\hline
\end{tabular}

depending on type of meeting (synchronous/asynchronous) or information exchanged (formal/informal).

The communication supports which seemed to be complementary for our needs were used during our study. They take into account our various means of exchange (spoken, written, drawn) and means of reception (vocal and visual). Our capacities to exchange concepts in suitable modes are implemented in these tools. Indeed, a draft is very natural and efficient to describe a geometrical structure, a text to clarify an equation or a vocal dialog to define concepts.

As the first experiment showed, with the forum as a communication media, asynchronous mode may lead to very specific exchanges. Like email communications [5], forum media can be of lean richness as it cannot contain instant feedback or visual cues. Despite this lack of richness, a forum owns other interesting properties. Actually, asynchronous media allows information storage and asynchronous work report. So, in this experiment, the forum was used as a storage site (like a PDM) for pre- and post-meetings, storing remarks, and saving key elements. These elements may be a mix of formal and informal communication (as the chat example of Fig. 13).

However, the asynchronous mode is not sufficient; it leads to a static design process and requires synchronization of the participants.

For the synchronous exchange mode, NetMeeting and Paltalk were used. The NetMeeting environment offers several tools: chat, whiteboard, application sharing, file exchange utilities, visual conference, and audio conference (but is restricted to only two persons).

In our meetings, there were more than two actors, so the visual and 
audio possibilities of NetMeeting were not sufficient. For our audio exchanges needs, Paltalk software was preferred. It allows audio and visual exchanges between several people along with a chat. For audio exchanges, only one person can speak at a time. The speaker locks out phone access for all the other actors when he speaks. This appeared to be of interest in exchange synchronization as will be illustrated later.

In an earlier study [24] some authors found that virtual team lack of richness makes it unfit to do new product developments. We think that a virtual team, as proposed in this study, needs synchronous meetings for new product development. These synchronous meetings were necessary for our design goal as they brought dynamics for group creativity and synchronization of individual works. Our exchanges during such virtual meetings were synchronous and mainly informal, due to the use of audio and visual media.

\section{Communication Media for Voice}

Support: Various communication modes have been highlighted, some of them imposed by internet communication technology restrictions.

A speaker wishing a "turn taking" system to be able to present information must use a tool such as a chat. However, the visual tools (chat, whiteboard, etc.) are numerous on the screen, and so, less powerful at holding human attention. Activation of the hearing sense is easier because this one is not disturbed by a lot of information. For this reason, the first meeting did not reach the expected goals. In meeting with video, turn taking is done by visual cues, but some studies showed that without these visual cues, turn taking is completely different [25].

During the second experiment, Paltalk was used as substitute for the vocal mode of NetMeeting.
By allowing a multispeaker mode with a controlled turn taking, undesirable group dynamics of the first meeting disappeared. This speech mode allowed good effectiveness during the third meeting, which corresponded to a project review. Indeed, under the control of one of the speakers, the meeting allowed us to sketch out our work and to answer important questions.

In spite of the success of this project review, we wonder if its effectiveness would remain valid during a creating phase (brainstorming). Indeed, a project review needs such a strict framework, for which hand catching mode seems very interesting. But, what was an asset for a project review can become a problem where a dynamic dialog is necessary.

More flexible tools do exist, such as "full duplex" technology, where turn taking can seem more anarchistic, but corresponding to dynamic interactions and a high media richness. Depending on the interaction between team members for creative phases, we may ask communication specialists, "how can communication technology best support free and open interactions?" [26].

Hardware: The codesign experiments highlight some problems due to the use of computer in support of communication. ICT has more or less the ability to answer our problems. It especially depends on which kind of information and communication are carried out. But it also depends on how peripheral hardware supports the interaction with the ICT.

Software functionalities can be controlled by means of common peripherals such as mouse or keyboard. Other peripherals may come to fit between the actor and his machine, to simulate natural communications. If present-day communication tools only act via our hearing and visual senses, the years to come will see interesting new interactions with force feedback hardware (haptic tools), like the ones used in distant medical surgery.

We tested the means of simulating the presence of each participant thanks to webcams, allowing us to capture video flow and to transfer it to other members. Video can be interesting to perceive reaction of the interlocutor, and give a more natural feel (which can be useful when collaborating directly with a client [9]). The contribution of video in our case may prove a good support when studied objects are of real world and are not exchangeable as data-processing entities. Indeed, it seems interesting to be able to make an experiment in real time, each participant being able to visualize what is going on.

During the creative processes of codesign, one may have to quickly detail a concept, for which no support was prepared. Within this framework of codesign, the sketches or diagrams are particularly adapted because designers are accustomed to this type of representation. This is why we used a graphics table on which the drawing is very user-friendly, very similar to a pencil and a sheet of paper. This peripheral does not bring new functionalities, but rather it supplements the drawing by more accessibility and ergonomics than the mouse device. Accessibility is a key point in such a communication structure [26] for hardware or software.

ICT may carry out informal exchanges, but their use must be managed to ensure a better collaborative communication process.

\section{Adapted Tools to Different Kinds of Collaborative Activities Undoubtedly, all} along the collaborative design process, there are different periods where communications are more or less intensive, involving a varying number of actors, and 
leading to more or less interactions and dynamics in the dialog, and to exchanges of more or less structured information. From our experiment, we asked ourselves which kind of ICT could best fit the needs of each communication context. With this in mind, we set up a basic typology of collaborative activities and tried to put aside a set of convenient communication tools.

\section{The "Brain Storming" Type}

Meeting: This kind of meeting is necessarily defined in a multipoint mode of communication because exchanges are the heart of this form of meeting. It essentially consists in building one or several potential solution(s) to a problem. Heavy calculations are not welcome during this phase. People discuss informally and then plan the actions to be taken in the form of tasks to carry out. With the result of this meeting, the attendees should have a clear idea of the continuations to give. The end of this meeting should thus lead to a list of tasks and information on the data useful to perform each of them.

The tools necessary for this type of exchanges are typically tools being very reactive and supporting informal exchanges. One can think of full duplex multipoint discussion and sights sharing (vocal and video channels). Notice that not providing video sharing could be a positive thing in brainstorming meetings as it might help people to express their ideas with less social pressure [5]. But there can also be technical contents which would rather be supported by a shared whiteboard (for sketches, screen shots, pictures, or 3-D models, along with some annotations) and a chat (for more textual dialog, where chronology of exchanges is important). Another issue in this context is to provide some support for the formalization of a report on the meeting contents and results (for example, in terms of objectives to be pursued). Of course, the meeting leader may write down some points in real time, but the ability of some ICT to store the contents of the exchanges it supported (whiteboard, chat, etc.) must also be used. Nevertheless, one difficulty may be to match time between different media so as to get the correspondence between a sketched proposal in the whiteboard and arguments in the chat contents, for example.

\section{The "Project Review" Type}

Meeting: This kind of meeting is also necessarily defined in a multipoint mode of communication because it is structured to inform the other collaborators of the course of the project. However, speaking time should be allocated to each major actor so that he presents his own results which must be reports prepared in advance and should not be built in the dynamics of the meeting. Computational simulations or technical analyses cannot be seriously considered simultaneously with this phase of the meeting. Undoubtedly, mechanisms supporting annotations must make it possible to write down the remarks of the other speakers. Each intervention in a review of the project aims to present the problems encountered by a specialist and his choices implying the community. Once the interventions of the participants are finished, an exchange must take place allowing a proposal for collective solutions. Exiting this phase, the participants should know the tasks they have to carry out.

Our experiments with this kind of meeting were a success because the unfolding of the exchanges were controlled by someone coordinating the meeting. Our choice of using a tool for oral exchange which does not support full-duplex (each speaker raises his hand and speaks when it is his turn) also had a very significant effect on the global efficiency by keeping people together and by compelling them to listen carefully to what the speaker was saying.
Often, one was having troubles understanding something in a presentation and tried to ask a question. But he had to wait for his turn to speak, and while waiting, he usually got his answer. By preventing the speaker from being interrupted, the meeting ran smoothly. On the other hand, the required concentration made it difficult to simultaneously take notes that would allow making an effective report and thus to have a clear documentation defining further phases of the project. Oral tools for dialog, text, or graph are indeed not memorized in the same way.

One of the participants, such as the leader of the presentation, should be in charge of writing down a report like in classical meetings. Thus, the report writer should be provided with some specific tools for this purpose. But we think that other participants should also be able to write down some information because they can be interested in specific technical information that is not relevant to the others. The form of these tools is to be discussed. If it appeared obvious that they should support text and pictures (like screen shots), they perhaps should also allow to record audio and video parts of the conference that are too rich to be summarized during the meeting.

The "Point-to-Point" Meeting: This type of meeting should allow fast interaction between a relatively limited number of participants (generally two) in order to be effective. Following a project review, the participants agreed on principles of the solutions. Then, while revisiting the details of their tasks, one of them discovers that he missed information and that he needs some explanation from one of his colleagues. A point-to-point meeting seems well suited to this synchronous collaborative phase. For example, these meetings are necessary to specify the values of the data useful to each task. These data may be built as shared 
concepts and parameters between the involved engineers. As these parameters are often related to ones available in dedicated technical software, a useful tool would provide a network connection between these shared concepts and the related technical parameters. This is one of the issues we propose in the final section of this paper.

One important and difficult point in these three kinds of meetings (synchronous) is to provide support for the formalization and capitalization of the exchanges, so that results, technical choices, and shared concepts emerging from these meetings can be used simultaneously with future asynchronous design steps.

Asynchronous Work: Of course, many tasks performed in an asynchronous mode are of great importance in the design project, and they need to be managed in a collaborative way. That is, important data exchanges may occur between the participants in such a phase.

This way of working has been well known for a few years in most industrial contexts. Related ICT are mainly Product Data Management systems used as technical information repositories, and forums for informal asynchronous exchanges. Although we used a repository and a forum throughout our experiment, we did not intensively study these points, because we decided to focus on synchronous communications. However, a key point we propose is to keep connected, even in asynchronous work, with the other engineers by way of shared concepts and parameters constructed in the synchronous mode, as defined in "point-to-point meetings."

\section{Adapted Tools to Intermediary} Objects Our experiments show that some tasks of design have a place in synchronous meetings. Particularly, exchanges of equations, parameters with their value, and meaning should be formalized.

The aim of this experiment was to establish formal links between our shared knowledge. Informal communications between mechanical engineers and electrical engineers lead to a common mental representation. This common representation can be exploited to formalize objects needed to the design of the structure.

The discussion about the spring is quite clear. Electrical engineers model the electromagnetic circuit with their own skills but parts of their models are associated with mechanical equations. A solution would have been to mix the knowledge needed to design the spring with the knowledge used to design electrical parts. This means that the spring equation can be parameterized enough to be used with the electrical model. This solution seems better than others in the case of a simple spring equation, but quickly becomes impractical: an electrical engineer cannot integrate the whole knowledge of every skill.

Examples of Exchanged

Information: In the air gap problem, not only drafts were exchanged, but also some simplified rules to explain choices. It seems that without them, solution delays happened. For example, the following rule can be applied several times during the design:

"suppress, or if it is not possible limit, the gap (e.g., the air-gap) between each part of the magnetic circuit."

Contrary to that, it is important for the mechanical designer to know that the permanent magnet can be anywhere in the magnetic circuit, but mechanically it is difficult. So, depending to its placement, its shape, and its mechanical stresses, it may be very different in shape, and so its cost may be increased or decreased. This is well known by the electrical designer and is easily discovered by the mechanical designer. In fact, this is common sense enriched by expert knowledge.

Designers must also coordinate their vocabulary (the clearance/air gap example). This corresponds to domain knowledge mapping. Such knowledge has been transmitted by text in chats, in the forum, or by voiced discussion in the net meeting.

As illustrated by the following figures (see Figs. 10-13), several kinds of information can be exchanged in the interprofessional dialog.

Schematic information was often used. This concerns:

Fig. 10. The principal figure (base element) for the dialog between the actors at the beginning of the design experiment: an example of schematic information exchange.

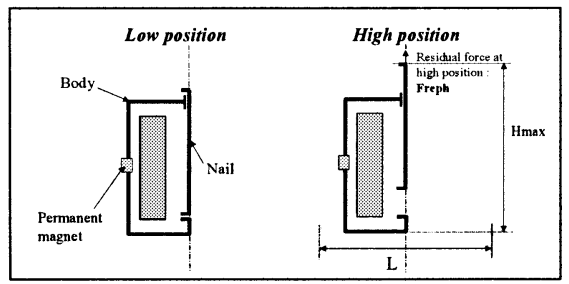

Fig. 11. Examples of figures drawn by the actors on the whiteboard with textual information and comments.

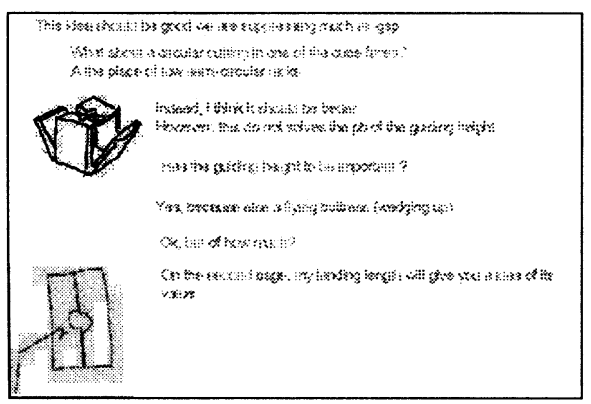

Fig. 12. Example of screen copies out of CAD software as shared on the whiteboard.

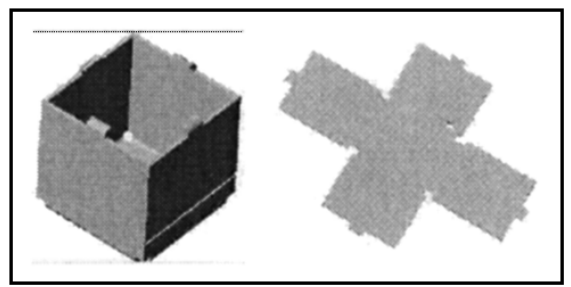


- the principle draft of the structure (see Fig. 10);

- drafts and sketches drawn by hand and digitized by a scanner or directly drawn by the mean of a graphic tablet (see Fig. 11);

- schemas, figures, or curves from $\mathrm{CAD}$ or analysis tools.

For Fig. 12, screen copies have often been put in the forum or exchanged via the whiteboard. Nongraphical information has also been exchanged:

- values in tables, professional rules (e.g., to define the exchange surface of the magnetic flux);

- equations with their semantics (see Fig. 13);

- parameters with their values, their units.

The Information Supports: During meetings, informal exchanges lead to the creation of a shared knowledge base. When both electrical and mechanical designers execute more formal processes, such as the sizing process, they may also solve some misunderstandings. These more formal processes require highly specific knowledge of scientific domains, which are hard to state clearly in a few words, and rely on dedicated tools to model the entire device. Although the concepts of each domain may be discussed by informal means, this phase requires more verbal exchanges between the participants. These exchanges are formal and need to be built dynamically. There is a need for a formalization of the concept of IO (intermediary objects) [15], [27].

A common database involves several possible scenarios:

Fig. 13. Example of textual information exchange through chat window.

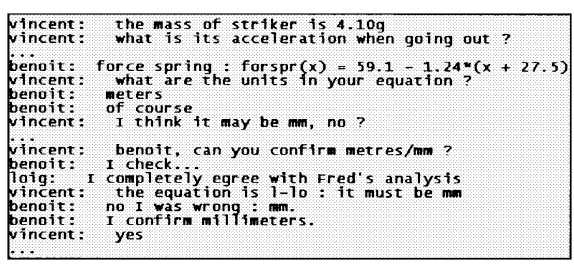

- In a first scenario, one may use simple ICT, that does not provide support for formalized IO. It leads to a nonoptimal design which must be improved.

- A second scenario would use dynamic exchanges of formalized IO between the designers. In the case of the discussion dealing with the spring, constraints were introduced by the electrical engineers in their models after consultation with mechanical engineers. One could imagine creating a link between the different actors providing them with up-to-date information about the spring (dimension, equation, etc.). These new IO increase the common model of each multidisciplinary designer.

- A third scenario considers a neutral designer in charge of the sizing. This designer must integrate enough knowledge from the different professions to do all the tasks alone. Moreover, every designer should be able to explain its knowledge once and to integrate it into a stand-alone container [19], [21]. In this way, every technical problem has to be defined in a global knowledge base, i.e., a product model. The neutral designer has only to consult this database to carry out the design. However, such global knowledge base would be hard to carry out.

Knowledge formalization is not a task to do once and for all, it is a highly dynamic process. The design process leads to the creation of IO built with the exchanges between designers during the process. The integration of a formalized support for IO during the design process to try to answer encountered problems should be supported by dynamic tools.

Communication tools are also information carriers. The exchanges between speakers are mainly informal; they are used to share ideas and to formalize concepts. When these concepts are formalized, they have to be clarified continuously. However, ICT are not always adapted to such a task.

During experiments, when exchanges are abundant, interesting information may be formalized in a textual way. For example, in our experiment, it has been carried out in the chat. At the end of the meeting, whiteboard and chat contents were backed up. After the meeting, the speakers with questions or comments put them into the forum. Several remarks can be made about this procedure relating to the re-exploitation of capitalized information during the meetings.

The utilization of the data created during the meetings is relatively complex. There is no link between information that appeared in various media, and it is hard to keep chronological consistency across the different communication tools. A tolerancing chain can indeed be expressed by an equation related to a diagram. However, the relation which was implicit during the meeting (thanks to the vocal support) does not exist in the backup. It is difficult to find both a temporal and relational links.

The practice of writing meeting reports comes mainly from the paper medium on which one can note successively the discussed items. During cooperative meetings, the significant number of ICT does not allow participants to take these necessary notes. Automatic mechanisms exist in centralized environments, such as CoCreate, making it possible to trace the actions carried out in the software. However, such an automatic mechanism cannot act as a filter as humans do when writing down notes. It seems necessary to formalize decision-makings by drafting of notes during the meeting.

What Kind of Exchange Supports for Structured Information : It appears from these collaborative 
design experiments that there are too few tools available supporting structured information exchanges in synchronous mode collaboration. Thus, we point out the need of such tools. They are dedicated to support the dynamic definition and structuring of data, information, or knowledge [28] in a collaborative design context, and we are looking for technologies that could support this.

New protocols are being developed to increase the internet communication possibilities. The XML (eXtensible Markup Language) formalism and the associated style sheets seem to be a good way to define, store, and exchange structured information. It is easy to understand and it offers numerous tools for automatic treatments. For example, it may allow the same piece of information to be presented in a different way according to the user's choice and points of interest. Also, the recent development of standards such as CORBA (Common Object Request Broker Architecture), DCOM (Distributed Common Object Model), and EJB (Enterprise Java Bean), for distributed objects and components, is useful to create connections between professional applications throughout heterogeneous computer sites and systems. By combining these technologies, it will be possible to develop new tools supporting dynamic structured information exchanges.

\section{New Collaborative Tools Proposal}

Specification Sheet: Cultural issues appear during collaborative work whenever at least two different skills are represented (electrical and mechanical in our case). Note that several subcategories of expertise may be distinguished in both of these technical fields. Both electrical and mechanical engineers work simultaneously and must share the results of their analyses. We have seen in the study that mechanical and electrical problems can appear together. It is quite impossible to enumerate every concept that they need to share. It mainly depends on the project context and must be defined dynamically. Therefore, one must provide the designers with a framework designed to manage synchronous meetings where concepts to be shared are dynamically defined. These tools must also support different levels and organizations of information. By referring to the taxonomy proposed in [27], one can speak about sharing and managing:

- "data," "information," or "knowledge";

- or from another point of view, "structured," "semistructured," or "nonstructured information";

- and a third classification may concern the "best" way of expressing the information: vocal, graphical (sketch, 3D model...) [28], textual, mathematical, rules, tables.

Thus, the ideal communication tool may support these different kinds of exchanges and provide designers with the ability to define simple intermediary objects, or more complex objects as an "aggregation" of basic ones.

Framework Proposal: Let us summarize now the framework of the new tools we are developing to support collaborative work in technical product design. A schematic view of the collaboration system framework can be found in Fig. 14.

Apart from tools dedicated to direct informal communication, such as chat, instant messengers, whiteboard, videoconference, or

Fig. 14. A schematic view of the collaboration framework.

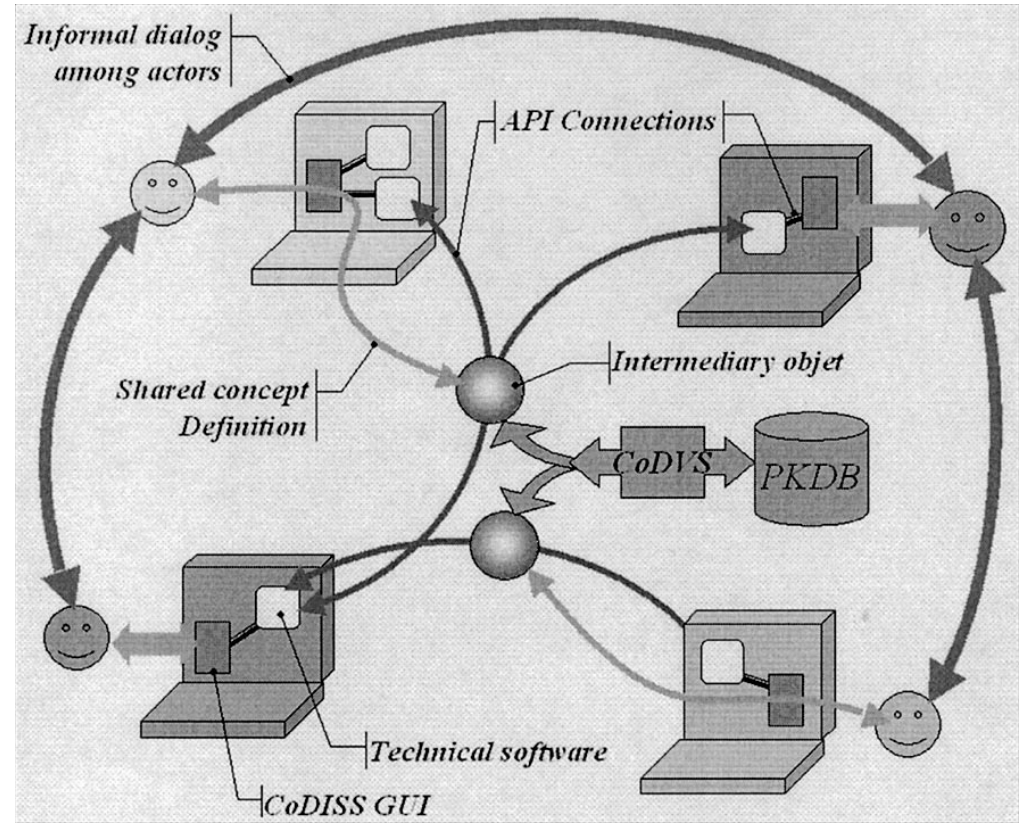


application sharing, one must provide every person involved in the design project with a GUI (graphical user interface) that allows the construction of IO to be shared with their colleagues. Then, connections with technical software are developed using each dedicated API (application programming interface): between the shared objects and some features or parameters in technical software, and between GUI and software. Among the latest generation of distributed objects development tools, we have chosen to manage shared information with the CORBA protocol. In this way, a tool called CoDISS (Cooperative Data \& Information Sharing System) has been developed to connect mechanical tools together. A system dedicated to store and manage shared objects, called CoDVS (Cooperative Data Versioning System) has been also developed. The aim of CoDVS is archiving and versioning of intermediary objects received from tools which can be connected to CoDISS.

A first mockup of this kind of software connections has been proposed in [29]. Note that more information about the development project of CoDISS and CoDVS can be found in [30].

Further Work: The issue of new tools supporting collaborative design over the internet is clearly to allow the emergence of a collaborative semantic community, a common project culture, or project world.

Thus, with this in mind, and as a matter of research prospects, we need to work on more precisely defining the specification sheet of these tools.

First, we must take an interest in other technical fields or cultures that may be quite different from mechanical and electrical engineering, fields where intermediary objects and knowledge cannot be formalized in terms of CAD model, schema, or equations, and where collective knowledge, action logic, or scale of values are very different. This will be done by getting in contact with several companies interested in collaborative design, for example in automotive, or aeronautical fields.

Second, we must work together with industrial sociologists and communication experts, to develop new skills in analyzing our further experiments and getting concrete proposals out of them. How does the culture of the designers relate to their use of communication media?

Finally, we must test other communication tools allowing more dynamics in dialogs and informal exchanges. This will allow the analysis of full duplex vocal communication, with or without turn-taking, to check the interest of multipoints video, etc. What are the effects of ICT tools on group dynamics during a collaborative design?

Simultaneously, we will continue the development of software mockup (CoDISS and CoDVS), to be able to test, as soon as possible in a real context, the new concepts we propose and that do not exist in present-day solutions.

\section{CONCLUSION}

This paper presents two different communities with their own specific semantic fields and domains which have been involved in the collaborative design of a technical product. Out of this experiment, we could extract some analysis of professional culture differences and confirm the need of building a common project knowledge. Intermediary objects are being developed at the interface between various technical worlds or professional cultures. Some analyses of adapted communication tools and organizations have also been proposed to better match the goals of various meeting contexts. The tools supporting online creation of this intermediary information must provide formalization facilities so as to increase data and information exchange efficiency. This may also increase the innovation ability of the company by providing more dynamics in communications between different actors involved in the design process.

\section{GLOSSARY}

API:

Application

Programming

Interface.

CAD/CAM: Computer-aided design, computer-aided manufacturing.

CoDISS: Cooperative data and information sharing system.

CoDvS: Cooperative data versioning system.

COBRA: Common Object Request Broker Architecture

CSCW: Computer-supported collaborative work

DCOM: $\quad$ Distributed Common Object Model

EJB: $\quad$ Enterprise Java Bean

EMP: Electromechanical plunger

FTP: $\quad$ File-transfer protocol.

GUI: Graphic user interface.

ICT: Internet communication tools.

IO: Intermediary objects

SCM: $\quad$ System (or software) configuration management.

XML: $\quad$ Extensible markup language.

\section{APPENDIX}

The Electromechanical Plunger: The electromechanical plunger (EMP) used to illustrate the paper is presented in [23]. Such a system presents numerous physical aspects: mechanical, magnetic, electrical, thermal. The power supply and the electronic control are not considered here.

The goal of the plunger is to hit another mechanism (a circuit breaker) which is going to cut 
an electrical circuit. In order to achieve this goal, a part called "striker" should move up and hit the other system. The energy is given by a spring which is held compressed until the plunger receives the order to release the "striker." At its low position, the striker is held down by magnetic force provided by a permanent magnet. To release the spring, the magnet force is canceled by electrically feeding a coil acting throughout a magnetic circuit. Then the spring quickly releases its energy, initiating the circuit breaker mechanism.

Numerical requirements of the specification sheet, needed to achieve the design, are as follows.

- Maximum bounding box in high position: $L=30 \mathrm{~mm}^{*} H_{\max }=40$ $\mathrm{mm}$.

- Minimal percussion energy:

$0.12 \mathrm{~J}$.

- Response time lower than 3.5 ms.

- Residual force in high position: $15 \mathrm{~N}$.

- Holding in low position: withstanding an acceleration of $2000 \mathrm{~m} \cdot \mathrm{s}^{-2}$.

- This product must be developed for mass production at low cost.

In the design of such a system, several technical tools are required, inducing several kinds of information exchanges between them.

In the following two subsections, we detail the tasks carried out by the mechanical engineer and by the electrical engineer.

Mechanical Engineer Tasks: In our project, the different mechanical fields were represented by three actors:

The designer deals with

- making technological choices,

- choosing materials,

- defining the original shape and thickness of the parts,

- fitting and tolerancing,

- performing kinematics simulations (collision, speed, energy).

The structural engineer is concerned with computations and simulations in

- mechanical strength (static and dynamic),

- deformation under load (elastic or plastic behavior),

- failure due to fatigue, buckling, large strain,

- vibration, modal analysis.

The manufacturing engineer deals with

- process planning,

- choice of manufacturing processes (machining, molding, stamping, welding. ..),

- simulations of specific processes,

- production cost estimation,

- deflection of the part while milling,

- tools trajectory computation,

- machining allowances.

Electrical Engineer Tasks: The selected following tasks are often done by the same electrical engineer [30], [32]:

- analysis of the plunger by fine modeling,

- macroscopic modeling for sizing processes,

- sizing process using optimization under constraints, [23],

- virtual prototyping to validate optimized structure.

In these tasks, the designer develops several models, taking or not taking into account the dynamic phenomena of the plunger.

To carry out these modeling, the electrical engineer uses several tools such as

- mathematical tools to build its analytical models,

- optimization tools,

- simulation tools for fine modeling to validate the analytical models developed for optimization.

\section{ACKNOWLEDGMENT}

The authors would like to thank Loig Allain, for his contribution to the two first NetMeeting experiments; Jean-Lou Barbet, Yvan Pradourat, Gregory Marin-Pache and Thomas Vuillet, students in DESS CCI (Joseph Fourier University, Grenoble), for coding the first tools CoDVS and CoDISS; the IPI institution for its financial support, and particularly Prof. Jan Ulijn for his encouragement and guidance.

\section{REFERENCES}

[1] N. Shyamsundar and R. Gadh, "Internet-based collaborative product design with assembly features and virtual design spaces," Computer-Aided Des., vol. 33, pp. 637-651, 2001.

[2] J. M. Ulijn and J. B. Strother, "Communicating in business and technology: from psycholinguistic theory to international practice," Peter Lang GmbH, Frankfurt, Germany.

[3] J. Ulijn and M. Weggeman, "Toward an innovation culture: What are its national, corporate, marketing and engineering aspects, some experimental evidence," in Handbook of Organizational Culture and Climate, C. Cooper, S. Cartwright, and C. Early, Eds. London, U.K.: Wiley, in press. 
[4] C. W. Chee, G. L. Harrison, J. McKinnon, and A. Wu, "Cultural influences on informal, information sharing in chinese and anglo-american organizations: An exploratory study," Accounting, Organiz. and Soc., vol. 24, no. 7, pp. 561-582, 1999.

[5] S. Kiesler and L. Sproull, "Group decision making and communication technology," Organiz., Behav. and Human Decision Processes, vol. 52, pp. 96-123, 1992.

[6] S. Mer, "Les mondes et les outils de la conception, pour une approche socio-technique de la conception de produit," Ph.D. dissertation, INPG, Grenoble, France, 1998.

[7] L. Karpik, "Les politiques et les logiques d'action dans la grande entreprise industrielle," Sociologie du Travail, vol. 1, 1972.

[8] L. Boltanski and L. Thévenot, De la Justification, les Economies de la Grandeur. Paris, France: Ed. Gallimard, 1991.

[9] A. P. D. van Luxemburg, J. Ulijn, and N. Amare, "The contribution of electronic communication media to the design process: Communicative and cultural implications," IEEE Trans. Prof. Commun., vol. 45, pp. 250-264, Dec. 2002.

[10] A. Xiao, H.-J. Choi, R. Kulkani, J. K. Allen, D. Rosen, and F. Mistree, "A web based distributed product realization environment," in Proc. DETC'01, ASME 2001 Design Engineering Technical Conf. and Computers and Information in Engineering Conf., DETC2001/CIE-21 766, Pittsburgh, PA, Sept. 9-12.

[11] V. Riboulet, "Co-Conception par Internet," in Mémoire de DEA 2001, INPG, Univ. Grenoble, France.

[12] M. Amiour, "Vers une Fédération de Composants Interopérables Pour Les Environnements Centrés Procédés Logiciels," Ph.D. dissertation, UJF-Grenoble I, Dept. Comput. Sci., Grenoble, France, June 17, 1999.

[13] B. S. Bell and S. W. J. Kozlowski, "A typology of virtual teams: Implications for effective leadership," Group and Organiz. Manag., vol. 27, no. 1, pp. 14-49, 2002.

[14] D. Xue and Y. Xu, "Web-based distributed system and database modeling for concurent design," in Proc. DETC'01, ASME 2001 Design Engineering Technical Conf. and Computers and Information in Engineering Conf., DETC2001/CIE-21 287, Pittsburgh, PA, Sept. 9-12, 2001.

[15] J.-F. Boujut and E. Blanco, "Intermediary objects as a means to foster co-operation in engineering design," in Proc. COOP'2000 Conf.-Workshop 2; The Role of Objects in Design Cooperation: Communication Through Physical or Virtual Objects, Sophia-Antipolis, France, May 2000, pp. 11-22.

[16] D. Vinck and A. Jeantet, "Mediating and commissioning objects in the sociotechnical process of product design: A conceptual approach," in Management and New Technology: Designs, Networks and Strategies. ser. COST Social Sciences, D. M. Lean, P. Saviotti, and D. Vinck, Eds: European Commission, 1995, ISBN 2-87 263-144-5. COST A3.

[17] J. Estublier, Distributed Objects for Concurrent Engineering (Lecture Notes in Computer Science). Berlin, Germany: Springer-Verlag, vol. 1675. SCM9, Toulouse, France, Sept. 5-7, 1999.

[18] S. E. Poltrock and G. Engelbeck, "Requirements for a virtual collocation environment," Information and Software Technology, vol. 1, no. 6, pp. 331-340, 1999.

[19] M. R. Cutkosky, R. S. Engelmore, R. E. Fikes, M. R. Genesereth, T. R. Gruber, W. S. Mark, J. M. Tenenbaum, and J. C. Weber, "PACT: An Experiment in Integrating Concurrent Engineering Systems," IEEE Computer, vol. 26, no. 1, pp. 28-37, 1993.

[20] L. Roucoules and S. Tichkiewitch, "CODE: A cooperation design environment. A new generation of CAD systems," Concurrent Eng. Res. and Applic., vol. 8, no. 4, pp. 264-280, 2000.

[21] L. Bannon and S. Bodker, "Constructing common information spaces,” in Proc. 5th Europ. Conf. Computer Supported Cooperative Work. Amsterdam, The Netherlands: Kluwer Academic, 1997, pp. 81-96.

[22] K. Schmidt and L. Bannon, “Taking CSCW seriously: Supporting articulation work," Computer Supported Cooperative Work, vol. 1, no. 1-2, pp. 7-40, 1992. 
[23] E. Atienza, F. Wurtz, J. Bigeon, M. Perrault, and V. Mazauric, “A methodology for the sizing and the optimization of an electromagnetic release," IEEE Trans. Magn., vol. 36, no. 4, pp. 1659-1663, 2000.

[24] A. Patti, J. Gilbert, and S. Hartman, "Physical co-location and the success of new product development projects," Eng. Manag. J., vol. 9, no. 3, 1997.

[25] S. Whittaker, "Rethinking video as a technology for interpersonal communications: Theory and design implications," Int. J. Human-Computer Studies, vol. 42, pp. 501-529, 1995.

[26] J. Carletta, A. Anderson, and R. McEwan, "The effects of multimedia communication technology on noncollocated teams: A case study," Ergonomics, vol. 43, no. 8, pp. 1237-1251, 2000.

[27] E. Blanco, O. Garro, D. Brissaud, and A. Jeantet, "Intermediary object in the context of distributed design CESA," in Computational Engineering in Systems Applications. Lille, France: IEEE-SMC, July 1996.

[28] M. Gardoni and E. Blanco, "Taxonomy of information and capitalization in a concurrent engineering context," Proc. 7th ISPE Int. Conf. Concurrent Engineering (CE'2000), 2000.

[29] B. Belhabib, F. Wurtz, J. Bigeon, and E. Atienza, "An integrated platform for electrical design,” in Proc. IEEE-IECON’99, San Jose, CA, Nov. 29-Dec. 3, 1999.

[30] V. Riboulet, B. Delinchant, L. Gerbaud, P. Marin, and F. Noël, "Tools for dynamic sharing of collaborative design information," in Recent Advances in Integrated Design and Manufacturing in Mechanical Engineering, G Gogu et al., Ed. Norwell, MA: Kluwer, 2002.

[31] B. Atienza, J. Bigeon, F. Wurtz, and V. Leconte, "EDEN: A generic integrated component software for constraint optimization," in Proc. 13th Conf. Computation of Electromagnetic Fields (Compumag 2001), Evian, France, July 2-5, 2001, pp. 232-233.

[32] F. Wurtz, J. Bigeon, and C. Poirson, "A methodology and a tool for the computer aided design with constraints of electrical devices," IEEE Trans. Magn., vol. 32, no. 3, pp. 1429-1432, 1996.

Benoit Delinchant holds the degree Electrical Engineer (ENSIEG 2000) and is preparing his Ph.D. at the Laboratoire d'Electrotechnique de Grenoble (LEG), France. His primary research interest is the integrated design environment of electrical systems.

Vincent Riboulet graduated from the École Normale Supérieure de Cachan (2001). $\mathrm{He}$ is lecturer in mechanical engineering at the University Joseph Fourrier. He is a Ph.D. student at the Laboratory 3S (Sols, Solides, Structures) in Grenoble, France, where he focuses on codesign by internet.

Laurent Gerbaud holds the degrees of Electrical Engineer (ENSIEG, 1988) and Ph.D. (1993) from the Institut National Polytechnique de Grenoble, France. He is a researcher at the Laboratoire d'Electrotechnique de Grenoble (LEG), where he studies the design process and tools of electrical systems.

Philippe Marin is associate professor in mechanical engineering at the ENSHMG. He received his Ph.D. degree from INPG in 1995. He works at the Laboratory 3S (Sols, Solides, Structures) in Grenoble, France, focusing on collaborative design and manufacturing processes. 
Frédéric Noël is associate professor in mechanical engineering at the Joseph Fourrier University, Grenoble, France. He received his Ph.D. degree from INPG in 1994. He works at the Laboratory $3 \mathrm{~S}$ (Sols, Solides, Structures) in Grenoble, France, where he focuses on finite elements simulation and collaborative design.

Frédéric Wurtz holds the degrees of Electrical Engineer (ENSIEG, 1993) and Ph.D. (1996) from the Institut National Polytechnique de Grenoble. He is a researcher at the Laboratoire d'Electrotechnique de Grenoble (LEG), where he works on the design process and tools of electrical systems. 